\title{
USUARIOS, MOTIVACIONES Y SOCIALIZACIÓN EN LOS PARQUES BIOSALUDABLES
}

\section{USERS, MOTIVATIONS AND SOCIALIZATION IN THE OUTDOOR HEALTH CIRCUITS}

\author{
López Capra, F. ${ }^{1}$ y Roca Cruz, A.1,2 \\ ${ }^{1}$ Federico López Capra. Graduado en Sociología por la Universidad de Granada. Granada, \\ España.felocapra@correo.ugr.es \\ 1,2 Antonio Roca Cruz. Profesor en la Facultad de Ciencias del Deporte de la Universidad de \\ Granada y Técnico Superior de Actividades Deportivas, Jefe de Sección del Servicio de Control \\ y Calidad de Servicios Deportivos en la Concejalía de Deportes del Ayuntamiento de Granada. \\ Granada, España. antonioroca@ugr.es
}

Código UNESCO: 631009 Calidad de vida

Clasificación Consejo de Europa: 16. Sociología del deporte

Recibido el 22 de marzo de 2018

Aceptado el 18 de junio de 2018

Correspondencia:

Federico López Capra

felocapra@correo.ugr.es

DOI: http://dx.doi.org/10.24310/riccafd.2018.v7i2.5087

\section{RESUMEN}

La rápida proliferación de los parques biosaludables motiva la presente investigación que pretende recoger información sobre los usuarios de estos espacios en la ciudad de Granada para conocer sus motivaciones y hábitos. En la producción de información se ha usado la encuesta empleando preguntas cerradas. La población objeto del estudio la constituyen los usuarios de estos espacios públicos y la muestra la componen 425 personas encuestadas en tres parques biosaludables. Los usuarios son en su mayoría personas mayores de 40 años, principalmente mujeres, aunque cada vez es mayor el número de usuarios con edades comprendidas entre los 18 y los 24 años. Respecto de las motivaciones, destaca su uso por razones de salud, para compensar el sedentarismo o el placer de realizar ejercicio físico.

Palabras clave: deporte, usuarios, espacio público, motivaciones. 


\section{ABSTRACT}

The rapid proliferation of the outdoor fitness parks drives to this research which is aimed at reporting information about the users of these spaces in the city of Granada in order to find out about their motivations and habits. When collecting the information, a survey with closed-ended questions was conducted to a sample of 425 users in three different parks. The majority of these users were people over 40 years old, mostly women. However, the number of users aged between 18 and 34 years old is on the rise. As for the most outstanding motivations, the parks are considered an option to improve health as well as to counteract the sedentary lifestyle or merely enjoy the pleasure of it.

Keywords: Sports, users, public space, motivations.

\section{INTRODUCCIÓN}

Con la puesta en marcha por parte del Ayuntamiento de Granada (España) del proyecto «Granada es Salud y Deporte» y siguiendo con la línea de trabajo iniciada en 2014 desde el Departamento de Sociología de la Universidad de Granada (UGR) centrada en el estudio de los parques biosaludables, se establecen lazos entre estas dos instituciones para llevar a cabo una investigación que satisfaga a ambas partes y que sirva de punto de partida para desarrollar un trabajo conjunto que permita comprender más y mejor a los usuarios de estos espacios, analizar sus opiniones y sugerencias de mejora para poder seguir adecuando los parque biosaludables a las necesidades cambiantes de un público que se sospecha diferente al que en sus inicios se esperaba atraer.

Los parques biosaludables surgieron en nuestro país hace algo más de una década bajo el apelativo de «parques geriátricos». Este tipo de denominación despeja cualquier tipo de duda sobre a quién estaban destinados estos espacios y quiénes se esperaba que fueran sus principales usuarios. En España, el primero de estos parques se construyó en Leganés (Madrid) en 2005 y no es de extrañar si se tiene en cuenta el público objetivo de estos espacios. En un estudio longitudinal iniciado en 1993, concluido en 2005 y publicado en $2006^{1}$, se calificó a los habitantes de este municipio como los más longevos de Europa; en ese mismo año y según datos del $\mathrm{INE}^{2}$, esta localidad madrileña contaba con un $11 \%$ de población mayor de 65 años (aproximadamente 21.000 de sus 180.000 habitantes). El progresivo aumento de la esperanza de vida entre sus habitantes hacía esperar que este porcentaje de personas fuera cada vez mayor. Por ello, las autoridades de este municipio, de acuerdo con las líneas de trabajo planteadas en el estudio citado previamente, asumieron la necesidad de implementar una serie de medidas que estuvieran dirigidas a mejorar la calidad de vida de las personas de la tercera edad. Una de las ideas más recurrentes recogidas a lo largo de la investigación era que «el envejecimiento saludable se define en base al buen funcionamiento físico y mental que, a su vez, incluye un número reducido de trastornos crónicos, buena movilidad, capacidad para llevar una vida independiente» ${ }^{3}$. Como respuesta a estos hechos, en Leganés se optó por construir el primer parque geriátrico de España que abriría la puerta a la difusión de estos espacios por todo el país. 
Durante los años 90, surgen diversas iniciativas y proyectos que comienzan a proponer al ejercicio físico como vía para paliar los efectos negativos del envejecimiento en diferentes países de Europa. Hopman-Rock y Van Hell ${ }^{3}$, en su estudio sobre la vejez en Holanda, ya advertían de los frutos en materia de salud que habían logrado los participantes en su investigación llevando a cabo ejercicio físico de baja intensidad. A nivel físico describen mejoras en el índice de masa corporal, reducción de la presión sistólica y disminución de la glucosa sanguínea entre otros beneficios. Diversos estudios, entre los que cabe destacar el de Liu et $\mathrm{al}^{4}$, apuntan a China como germen de la implantación de medidas para contrarrestar los efectos negativos del envejecimiento a través del deporte. Según los resultados arrojados por las investigaciones llevadas a cabo en la materia y en consonancia con una larga tradición de realizar actividades físico deportivas en los espacios públicos, se han venido desarrollando en el país asiático, pionero en el cuidado de la salud a través del ejercicio físico, diversas iniciativas de fomento de actividades al aire libre que pretenden unificar los conceptos de salud y ocio al mismo tiempo que ofrecen a las personas de la tercera edad espacios donde poder desarrollar ejercicio físico de forma gratuita y continuada. Allí nacen los primeros «parques geriátricos» y de investigaciones más recientes se desprende que sigue siendo una prioridad fundamental en materia de salud el desarrollo de estrategias de intervención que contribuyan a promover el ejercicio físico en el tiempo libre, con mayor énfasis en zonas urbanas ${ }^{5}$. Estos nuevos espacios públicos de uso gratuito pronto comenzaron su expansión por varios países del norte y este de Europa hasta llegar hace algo más de una década a España, donde su difusión ha sido rápida y continuada.

Si bien en Leganés los mayores de 65 años representaban un 11\% de la población en el año 2005, según datos del INE $^{2}$ en Granada este grupo de población representaba un 17,6\% a la misma fecha. En la actualidad, las personas mayores de 65 años suponen aproximadamente un $20 \%$ del total de población de Granada y en los últimos 10 años, solo en el área metropolitana de Granada, se han construido 60 parques de este tipo. Según Rodríguez et al. ${ }^{6}$, el desarrollo y difusión de estos espacios deportivos se produce porque en la nueva situación demográfica:

«se plantea el reto de hacer llegar a todas las personas mayores un plan de prevención y tratamiento del deterioro musculoesquelético que se produce a través de ejercicios de coordinación, equilibrio, movilidad y fuerza dirigidos especialmente a ellos».

A pesar de que a día de hoy parece algo obvio, durante muchos años, el fomento de la actividad física en pos de alcanzar una mejor salud en la tercera edad se veía como algo poco útil y demasiado costoso debido principalmente a la resistencia que se le presupone a este grupo de edad a la asunción de nuevos hábitos y conductas en temas relacionados con el cuidado y la salud. Según Giró ${ }^{7}$, el cambio más importante en este sentido se da en el momento en el que empieza a asumirse como necesario un plan de prevención de la enfermedad en la tercera edad a través del fomento de la actividad física. Este plan se centrará principalmente en el mantenimiento de las capacidades funcionales y motoras 
que conllevan un importante freno al deterioro mental y físico del anciano. Será este caldo de cultivo en el que iniciativas como la del parque biosaludable, otrora parque geriátrico, serán vistas como elementos importantes de lucha contra el menoscabo físico de las personas mayores a la par que se convierten en una vía de socialización y de mantenimiento de relaciones interpersonales. Tanto es así que, según la información que el propio Ayuntamiento de Granada ${ }^{8}$ ofrece en su página web, estos 60 parques instalados por todo el municipio y que cuentan con 527 aparatos biosaludables están orientados principalmente a que adultos a partir de los 40 años y principalmente mayores de 60 lleven a cabo una práctica del ejercicio físico accesible y gratuita que les permita mejorar su movilidad, flexibilidad, resistencia y tonificar su cuerpo. Estas iniciativas orientadas principalmente a la tercera edad se ven legitimadas por un discurso político que tiene un doble objetivo. Por un lado, la tercera edad constituye un nutrido sector de la población cada vez más amplio. Consumidores de bienes y servicios y potenciales votantes que encuentran en estos espacios la oportunidad de desarrollar una actividad física de forma habitual y sin coste ni horarios establecidos, lo que supone un importante aliciente. Por otro lado, se trata de un creciente grupo de ciudadanos causantes de importantes gastos en materia de salud ligados en gran medida al sedentarismo y la soledad ${ }^{9}$. Por ello, iniciativas como las del parque biosaludable cumplen una doble función para sus impulsores; a la par que contrarrestan aspectos negativos del envejecimiento a través del fomento del ejercicio físico y las relaciones sociales, mejorando así la salud tanto física como mental de sus usuarios y reduciendo el gasto en sanidad, aparecen como una inversión destinada preferentemente a un sector concreto de la población, la tercera edad, que, a diferencia de otros grupos de edad como los niños o los adolescentes, constituyen un objetivo electoral con gran peso político y demográfico ${ }^{10}$.

Los parques biosaludables, a pesar de ser espacios destinado en sus orígenes a un espectro concreto de la población como es la tercera edad, con el paso del tiempo se han ido abriendo a nuevos usuarios y prácticas deportivas y también su nomenclatura se ha ido adaptando puesto que empezaron denominándose como «parques geriátricos». Asimismo, el tipo de maquinaria que encontramos en ellos ha variado dando cabida a otro tipo de actividades que permiten no solo el mantenimiento del sistema musculoesquelético, la resistencia cardiovascular o el masaje, sino también el desarrollo de habilidades físicas como la flexibilidad, la musculación o la práctica de calistenia. Esta nueva oferta de actividades físicas atrae a otros segmentos de la población y amplía el abanico de edades de los potenciales usuarios de estos espacios. En su estudio «La socialización de las personas mayores en el parque biosaludable», Hita et al. ${ }^{11}$ advertían de que en Granada se evidencian casos de «invasión» de estos espacios por parte de otros grupos de edades y de la «convivencia» que aquí se genera. 


\section{MÉTODO}

La presente investigación, un estudio exploratorio sobre el uso de los parques biosaludables del municipio de Granada, se ha llevado a cabo empleando exclusivamente metodología cuantitativa. Como técnica de producción de información se ha optado por la encuesta empleando preguntas cerradas. La población objeto del estudio la constituyen los usuarios de los parques biosaludables del municipio de Granada. La muestra la componen 425 personas encuestadas en tres parques biosaludables escogidos por estar situados en barrios con una ubicación estratégica en el norte, centro y sur de la ciudad de Granada (Joaquina Eguaras, parque Federico García Lorca y parque Bola de Oro) lo que permite, además de una diferenciación geográfica, una diferenciación sociodemográfica por la propia composición de los residentes de cada uno de estos barrios. El primero de estos parques, el de Joaquina Eguaras, se sitúa en la zona Norte de Granada en la que, según Palomares ${ }^{12}$, predominan las clases populares o clases obreras; en general acoge a personas de mediobajo poder adquisitivo. El segundo de estos parques se ubica el distrito CentroRonda de Granada considerada por Palomares una zona de predomino burgués, es decir, de clase alta o media-alta. El último de los tres parques analizados se enclava dentro del parque de Bola de Oro, perteneciente al distrito Genil, barrio en el que, según Palomares, predomina una caracterización social de tipo medio o medio-alto. De este modo, con el estudio de estos tres parques, aunque con limitaciones, podremos tener una buena perspectiva de análisis de los parques biosaludables de la ciudad de Granada.

Tabla 1. Distribución de las encuestas

\begin{tabular}{|l|r|r|}
\hline Parque & Frecuencia & Porcentaje \\
\hline $\begin{array}{l}\text { Joaquina } \\
\text { Eguaras }\end{array}$ & 118 & 27,8 \\
$\begin{array}{l}\text { García } \\
\text { Lorca }\end{array}$ & 156 & 36,7 \\
$\begin{array}{l}\text { Bola de } \\
\text { Oro }\end{array}$ & 151 & 35,5 \\
Total & 425 & 100,0 \\
\hline
\end{tabular}

La distribución de las 425 personas encuestadas en los parques biosaludables queda reflejada en la Tabla 1. Como vemos, la representación en los parques Federico García Lorca y Bola de Oro es bastante similar, quedando el parque Joaquina Eguaras levemente infrarrepresentado.

Para evitar posibles sesgos en cuanto a horarios o días de la semana se han cumplido cuotas de encuestas durante una semana completa (7 días de lunes a domingo) en horario de mañana y tarde en cada uno de los tres parques.

Al entrar a analizar las motivaciones de los usuarios de los parques biosaludables, además de un análisis de frecuencia y de cruzar las motivaciones 
con diversas variables sociodemográficas, se llevó a cabo un análisis factorial exploratorio que permitió agrupar las variables que presentaban una significación común. Este tipo de análisis permite simplificar y reducir las motivaciones de quienes acuden al parque biosaludable según la correlación que presentan sus resultados logrando así obtener diferentes perfiles de usuarios.

\section{RESULTADOS}

Dentro de estos parques encontraremos dos perfiles de usuario que predominan sobre el resto. El primero de ellos, a diferencia de lo que planteado en su día por García Ferrando ${ }^{13}$, lo componen personas de más de 40 años, sin distinción por sexo y que han cursado estudios secundarios o superiores. En cuanto a su situación laboral, en su mayoría son trabajadores o jubilados. Las personas que encajan en este perfil generalmente viven acompañadas, acuden al parque mayoritariamente en horario de mañana o a últimas horas de la tarde y lo hacen en solitario. Tendrán frecuentes interacciones con otros usuarios de las que una de cada cinco se mantendrá fuera de los límites del parque, generalmente para actividades de ocio. En cuanto a la frecuencia semanal de uso, este perfil de usuario acude al parque de cuatro días o más y lo usa desde hace más de dos años. Aparece también una tendencia a abandonar otros espacios de actividad deportiva en beneficio del parque biosaludable, sobre todo gimnasios de pago y por causas económicas. El otro perfil destacable lo componen los usuarios «invasores» ${ }^{11}$. Se trata de personas jóvenes, menores de 40 años en general y mayoritariamente menores de 25. Dentro de este perfil seis de cada 10 usuarios serán mujeres. En cuanto al nivel de estudios, ocho de cada diez han cursado o está cursando estudios superiores. Sobre su situación laboral, la mitad estudian y un tercio de ellos trabajan. Las personas que encajan en este perfil hacen uso del parque mayoritariamente durante la tarde y desde hace menos de un año. En su mayoría viven acompañados y dos de cada cinco no serán originarias de Granada, sino que tendrán su residencia habitual en otra ciudad. Las interacciones que puedan esperarse entre personas con este perfil y otros usuarios del parque, como ya apuntaban Hita, Martos y Romero ${ }^{11}$ son escasas y el hecho de mantener éstas fuera del espacio biosaludable parece bastante improbable.

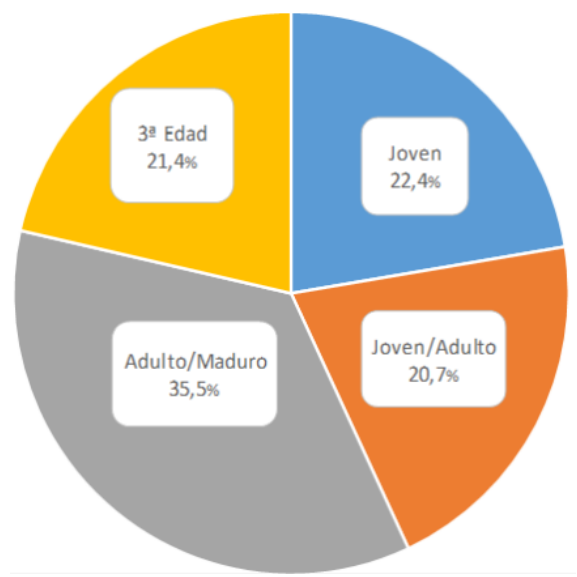

Gráfico 1 Distribución de los encuestados por edad 
En la línea de lo planteado en el artículo citado previamente, el parque biosaludable parece haber abierto sus puertas a otros usuarios diferentes a sus destinatarios principales. La posible convivencia intergeneracional que podría esperarse, atendiendo a los diferentes horarios de uso de cada grupo de edad, parece no materializarse.

En cuanto a las motivaciones para el uso de los parques biosaludables observamos que el $86,8 \%$ de los encuestados manifiesta que acude al parque biosaludable para mejorar su salud y aspecto físico, siendo esta la motivación que presenta el porcentaje más elevado de los encontrados. Un 70,8\% de los encuestados tiene como motivación principal para acudir a los parques biosaludables el tratar de compensar el sedentarismo de la vida cotidiana mientras que solo un $23,3 \%$ acude al parque por prescripción médica. El desarrollo de nuevas habilidades físicas presenta niveles parejos de acuerdo $(44,2 \%)$ y desacuerdo (37,2\%). Las motivaciones profesionales arrojan el nivel más alto de desacuerdo con sólo un $8,5 \%$ de aceptación por parte de los encuestados. Un $65,6 \%$ de los usuarios manifiesta que el placer de practicar ejercicio físico es una motivación importante para ellos y la interacción social, aunque con mayoría de desacuerdo, presenta un alto porcentaje de importancia como motivación secundaria para el uso de estos espacios.

En la investigación se proponen las siete posibles motivaciones expuestas previamente. Con la finalidad de tratar de establecer un perfil 0 perfiles de usuarios de los parques se ha llevado a cabo un análisis factorial tratando de agrupar aquellas que se correlacionan y simplificándolas hasta obtener posibles perfiles de usuarios. La matriz de componente rotado obtenida se presenta en la Tabla 2.

Tabla 2. Matriz de componente rotado. Análisis factorial

\begin{tabular}{|l|l|l|l|l|}
\hline & 1 & 2 & 3 & 4 \\
\hline $\begin{array}{l}\text { Por el placer de } \\
\text { practicar ejercicio físico }\end{array}$ &, 786 & & & \\
\hline $\begin{array}{l}\text { Por prescripción } \\
\text { médica }\end{array}$ &,- 751 & & & \\
\hline $\begin{array}{l}\text { Mejorar salud y aspecto } \\
\text { físico }\end{array}$ & &, 766 & & \\
\hline $\begin{array}{l}\text { Para compensar la } \\
\text { inactividad de la vida } \\
\text { cotidiana }\end{array}$ & &, 742 & & \\
\hline $\begin{array}{l}\text { Por motivos } \\
\text { profesionales }\end{array}$ & & &, 825 & \\
\hline $\begin{array}{l}\text { Para desarrollar nuevas } \\
\text { habilidades físicas }\end{array}$ &, 379 & &, 661 & \\
\hline $\begin{array}{l}\text { Para relacionarme y } \\
\text { conocer gente }\end{array}$ & & & & \\
\hline $\begin{array}{l}\text { a. La rotación ha } \\
\text { convergido en 6 } \\
\text { iteraciones. }\end{array}$ & & & & \\
\hline
\end{tabular}


Una vez analizados los datos se han creado cinco perfiles de práctica deportiva diferentes en relación con las motivaciones de quien la lleva a cabo. Los perfiles propuestos son los siguientes:

- En primer lugar, tenemos la «Práctica deportiva lúdica» que es propia de usuarios cuyas motivaciones principales son el placer de practicar ejercicio físico y la posibilidad de desarrollar nuevas habilidades físicas. Este perfil de práctica deportiva estará confrontado el de quienes acuden al parque biosaludable por prescripción médica, usuarios que practican una «Práctica deportiva médica».

- Encontramos otro perfil de usuarios cuyas motivaciones principales son la mejora de su salud y aspecto físico y la posibilidad de compensar el sedentarismo. Esta agrupación de motivaciones se llamará «Práctica deportiva saludable».

- Otro perfil de usuario será el que esté motivado por causas profesionales y por la posibilidad de desarrollar nuevas habilidades físicas. Este perfil de motivaciones será la «Práctica deportiva de entrenamiento».

- Por último, encontramos el perfil de usuario que acude al parque biosaludable motivado por la posibilidad de establecer interacciones sociales. Recibirá el nombre de «Práctica deportiva social».

Ateniéndonos a las motivaciones, los usuarios de los espacios biosaludables no presentan diferencias por sexo. Buscan, en su mayoría, mantener o mejorar su salud y aspecto físico, compensar la inactividad cotidiana y practicar ejercicio físico por placer. Si el usuario es joven, su motivación principal es mejorar su salud y estado de forma física practicando deporte por placer y tratando de desarrollar nuevas habilidades físicas. Si se trata de un usuario joven/adulto, tendrá motivaciones similares al anterior y a éstas hay que añadir la prevención del sedentarismo y la posibilidad de relacionarse con otros usuarios, que en este rango de edad empieza a cobrar relevancia. Entre los adultos/maduros el hecho de poder relacionarse pierde importancia, a pesar de ser el grupo de edad que presenta mayor número de interacciones y las prescripciones médicas empiezan a ser relevantes. Los principales motivos de este grupo de edad para usar el parque son mejorar su salud y compensar la inactividad. Por último, la tercera edad acude a los parques principalmente por salud y para combatir el sedentarismo; motivaciones como las prescripciones médicas y la posibilidad de relacionarse con otros usuarios pasan a un primer plano cobrando más importancia que en el resto de segmentos de edad.

El análisis revela que más de la mitad de los usuarios que no han apreciado mejoría en su estado de salud desde que acuden a los parques biosaludables siguen haciendo uso de ellos después de más de dos años. Si exceptuamos a su vez a quienes acuden por prescripción médica, de nuevo, algo más de la mitad de estos usuarios continúan acudiendo a los parques y, en su mayoría, son personas de más de 65 . Como apuntan del Valle y Coll ${ }^{14}$ «las relaciones interpersonales impulsan la participación social de las personas mayores y son parte activa en su envejecimiento». Por ello cabe pensar que, a 
pesar de la poca probabilidad de establecimiento de relaciones intergeneracionales, la posibilidad de relacionarse con otros usuarios con características afines o de establecer relaciones más duraderas condicionan el sostenimiento de la práctica deportiva en los parques biosaludables. Es por esto que nos parece interesante indagar sobre el papel de la interacción social.

Según los análisis, se puede afirmar que ésta cobra mayor importancia a medida que aumenta la edad de los usuarios de los parques biosaludables. Los mayores, además de ser quienes más importancia le dan a ésta entre sus motivaciones, son quienes más interactúan dentro de los límites del parque y los que, con mayor frecuencia, exportan las relaciones sociales fuera éste para realizar principalmente actividades de ocio. Si nos limitamos a la interacción verbal, el grupo de los adultos/maduros presenta valores más elevados que el resto; esto puede hacer pensar que el hecho de encontrarse aún en edades intermedias facilita su interacción tanto con jóvenes como con personas mayores que ellos. Por su parte, en las personas de la tercera edad, con la paulatina pérdida de capacidades que acarrean problemas de autoestima, distracción 0 pérdida de facultades sociales ${ }^{15}$, se dificultan este tipo de interacciones a pesar del interés que puedan tener en ellas, que se ha visto que es elevado.

Ahondando en las circunstancias que pueden dificultar la interacción social con desconocidos dentro del parque biosaludable se propone el acudir a éstos acompañado. Aunque se presupone que existirán interrelaciones personales entre el usuario y sus acompañantes, el análisis revela que, en efecto, el establecimiento de nuevas relaciones personales con otros usuarios se ve condicionado por este hecho. Dos terceras partes de las interacciones acaecidas en los parques biosaludables las protagonizan personas que acuden a ellos en solitario.

\section{CONCLUSIONES}

Dentro de los parques biosaludables de la ciudad de Granada podemos distinguir dos perfiles principales de usuarios. El primero de ellos, al que nos permitimos denominar de «usuarios originales», está compuesto por personas mayores de 40 años, en su mayoría trabajadores o jubilados y que acuden al parque más de 4 veces a la semana y aproximadamente el $80 \%$ de ellos dice interactuar con otros usuarios dentro del espacio del parque biosaludable. El otro perfil, denominado de «usuarios invasores», está compuesto principalmente por personas entre los 18 y los 24 años de edad, principalmente mujeres. La mayoría estudia y acude al parque menos de 4 veces a la semana. Dentro de este perfil, el nivel de interacción social dentro del parque es muy inferior al del perfil anterior ya que asciende escasamente al $20 \%$. En cuanto a la interacción no hemos podido constatar un aumento de las relaciones intergeneracionales dentro de los parques biosaludables.

Las motivaciones principales de los usuarios son la mejora de la salud y el aspecto físico, la lucha contra el sedentarismo y el placer por practicar ejercicio físico. Estas motivaciones nos permiten crear cinco tipos de práctica deportiva 
como son la lúdica, la médica, la saludable, la de entrenamiento y la social en función de qué busca encontrar cada usuario cuando acude a los parques biosaludables.

Entre los distintos grupos de edad, sobre todo entre los mayores, la interacción dentro de los parques biosaludables es elevada. Entre estos últimos, además, constituye la principal motivación de uso de estos espacios.

\section{REFERENCIAS}

1. Zunzúnegui M., Otero-Piume Á., Béland F. y Rodríguez-Laso Á. Relaciones Sociales y envejecimiento saludable. Bilbao: FUNDACIÓN BBVA. 2006

2. INE. Instituto Nacional de Estadística. [Online].; 2017. Disponible en:http://www.ine.es/dyngs//NEbase/es/categoria.htm?c=Estadistica_P\&cid=12 54734710990.

3. Hopman-Rock M. y Van Hell L. Los efectos de un programa de ejercicio de baja intensidad en mayores de 75 a 85 años que viven en comunidad. En Ortega Meléndez A. Actividad física y salud en la tercera edad. Madrid: Instituto Nacional de Servicios Sociales; 1996: 247-248.

4. Liu Z., Speed S. y Beaver K. Perceptions and attitudes towards exercise among Chinese elders - the implications of culturally based self-management strategies for effective health-related help seeking and person-centred care. Health Expect. 2015; 18: 262-272.

5. Muntner P., Gu D., Wildman R. P., Chen J., Qan W., Whelton P. K., \& He J. Prevalence of Physical Activity Among Chinese Adults: Results From the International Collaborative Study of Cardiovascular Disease in Asia. American Journal of Public Health. 2005; 95: 1631-1636.

6. Rodríguez C., Sáez C., y López R. El parque geriátrico: fisioterapia para nuestros mayores. Revista Gerokomos. 2007;18: 84-88.

7. Giró-Miranda J. Envejecimiento y sociedad, una perspectiva pluridisciplinar. La Rioja: Servicio Publicaciones Universidad de la Rioja. 2004.

8. Ayuntamiento de Granada. Concejalía de Medio Ambiente y Mantenimiento. [Online]; $2016 . \quad$ Disponible en: http://www.granada.es/inet/mantenimiento.nsf/xbiosaludable/10D62C7435650C C4C125784000251888.

9. E. Estudio de los circuitos biosaludables para la tercera edad en España. Revista Internacional de Medicina y Ciencias de la Actividad Física y el Deporte. 2009;9: 25-38.

10. Gil E. El poder gris: una nueva forma de entender la vejez. Barcelona: Mondadori. 2003.

11. Hita C., Martos P. y Romero, A. La socialización de las personas mayores en el parque biosaludable. Revista Iberoamericana de Ciencias de la Actividad Física y el Deporte. 2015: 21-33.

12. Palomares-Linares I. Movilidad residencial, sedentarismo y estructura social urbana: La ciudad metropolitana de Granada. Granada: Universidad de Granada. 2013.

13. García-Ferrando M. Aspectos sociales del deporte. Madrid: Alianza Editorial. 1990. 
14. Del Valle G. y Coll L. Envejecimiento saludable. Barcelona: Universitat Autònoma de Barcelona. 2011.

15. Barrios Duarte René, Borges Mojaiber Rolando, Cardoso Pérez Laura del Carmen. Beneficios percibidos por adultos mayores incorporados al ejercicio. Rev Cubana Med Gen Integr [Internet]. 2003 Abr [citado 2018 Jul 12] ; 19( 2 ): . Disponible en: $\quad$ http://scielo.sld.cu/scielo.php?script=sci_arttext\&pid=S0864$21252003000200007 \&$ Ing=es.

Referencias totales citadas: 15

Referencias citadas correspondientes a la RICCAFD: 1 